\title{
ON THE WEAK-COUPLING LIMIT AND COMPLETE POSITIVITY
}

\author{
F. Benatti \\ Dipartimento di Fisica Teorica, Università di Trieste \\ Strada Costiera 11, 34014 Trieste, Italy \\ and \\ Istituto Nazionale di Fisica Nucleare, Sezione di Trieste \\ R. Floreanini \\ Istituto Nazionale di Fisica Nucleare, Sezione di Trieste \\ Dipartimento di Fisica Teorica, Università di Trieste \\ Strada Costiera 11, 34014 Trieste, Italy
}

\begin{abstract}
We consider two non-interacting systems embedded in a heat bath. If they remain dynamically independent, physical inconsistencies are avoided only if the single-system reduced dynamics is completely positive also beyond the weak-coupling limit.
\end{abstract}




\section{Introduction}

In a variety of different contexts, ranging from quantum optics to the foundations of quantum mechanics, the dissipative and irreversible time-evolutions of open quantum systems in weak interaction with suitable, large environments are commonly described by the so-called quantum dynamical semigroups [1-3]. These consist of linear maps $\Lambda_{t}, t \geq 0$, that act on the physical states (density matrices) $\rho^{S}$ of the open systems, $S$, and satisfy an evolution equation of Kossakowski-Lindlab form [4-10].

The maps $\Lambda_{t}$ are linear and completely positive, that is, if $S$ is coupled to an arbitrary $N$-level system $S_{N}$, the maps $\Lambda_{t} \otimes \mathrm{id}_{N}$ preserve the positivity of the states $\rho^{S+S_{N}}$ of the compound system $S+S_{N}$ for all $N$.

Complete positivity is an algebraic property [10] whose physical implications are better understood in the negative [11]: if $\Lambda_{t}$ is not completely positive, then for some $N$ an entangled initial state of $S+S_{N}$ surely exists which develops negative eigenvalues under the action of $\Lambda_{t} \otimes \mathrm{id}_{N}$. On the other hand, if the initial state of $S+S_{N}$ is not entangled, i.e. $\rho^{S+S_{N}}=\rho^{S} \otimes \rho^{S_{N}}$, it never develops negative eigenvalues under the action of $\Lambda_{t} \otimes \operatorname{id}_{N}$.

Physical consistency demands that the eigenvalues of $\Lambda_{t}\left[\rho^{S}\right]$ must be positive in order to be interpretable as probabilities. It is logically necessary, but physically less compulsory, that the same should be true of all $\Lambda_{t} \otimes \operatorname{id}_{N}\left[\rho^{S+S_{N}}\right]$; this latter request would only be guaranteed by the complete positivity of $\Lambda_{t}$. Yet, the system $S_{N}$ is totally arbitrary and unchanging, only statistical correlations with $S$ being allowed. Although such an occurrence is always possible, it is not always accepted [12] as a justification [11] of complete positivity as a necessary property of reduced dynamics.

It is sometimes argued [12-14] that complete positivity is the consequence of two auxiliary technical simplifications that are essential in the standard [1-3] derivation of quantum dynamical semigroups from the closed dynamics of the system $S$ plus its environment. It is in fact assumed $a$ ) that the initial state of $S$ be uncorrelated to that of the environment, and $b$ ) that a Markov approximation is possible on rescaled times $\tau=\lambda^{2} t$, where $\lambda<<1$ is the strength of the system-environment interaction [2-9] (the weak-coupling limit). However, requests $a$ ) ad $b$ ) are not always physically plausible [12-21]; in particular, it might be necessary to examine the subsystem dynamics on times of the order of $\lambda^{4} t$, hence beyond the weak-coupling limit $[15,17-20]$ and, in such instances, dynamics not of completely positive type may appear.

The standard derivation of the Redfield-Bloch equations [22], commonly used to describe the reduced dynamics of open two-level systems in chemical physics, fails to produce even positive dynamical maps $\Lambda_{t}$ [4]. While the danger is fully acknowledged [16], it is not accepted that one should end up with a completely positive time-evolution [4]. Rather, it is argued that only those states whose positivity is preserved should be physically admissible or that a slippage in the initial conditions is needed in order to avoid inconsistencies [13-17]. However, from the previous considerations, it is clear that accommodating the problem of positivity does not properly address the issue of complete positivity which is strictly related to quantum entanglement.

The relevance and role of complete positivity is most clearly seen in the phenomenology of neutral K-mesons as open quantum systems in interaction with a gravitational background. Geometrical fluctuations at Planck's scale act as a source of dissipation and 
decoherence [23-29]. Each single K-meson is thus assumed to evolve according to a semigroup of positivity-preserving, entropy increasing phenomenological linear maps $\Lambda_{t}$. It turns out [25-29] that these maps must also be complete positive. Otherwise, physical inconsistencies would plague the resulting phenomenology of couples of K-mesons evolving in time according to the factorized dynamical maps $\Lambda_{t} \otimes \Lambda_{t}$. More important, these dissipative phenomenological models can actually be put to test in experiments performed at the so-called $\phi$-factories $[26,29]$.

In the following, we show that if the environment is such that there is no induced interaction between two otherwise non-interacting systems embedded in it, then the twosystem reduced dynamics is in factorized form. Moreover this will be true at the 2-nd and 4-th order in the system-environment coupling constant $\lambda$. It then follows that if, beyond the weak-coupling limit, the reduced dynamics is not completely positive, then either the environment establishes a dynamical dependence between the two subsystems or the approximations leading to the reduced dynamics are not physically consistent.

\section{Complete Positivity.}

Let $S$ be a physical system whose time-evolution is given by a (semi)group of linear maps $\Lambda_{t}$ acting on the states of $S$ represented by density matrices $\rho^{S}$. Usually, $\Lambda_{t}$ is positive and thus preserves the positivity of the eigenvalues of $\rho^{S}$. ¿From an abstract point of view $[10,11], \Lambda_{t}$ is completely positive if and only if the map $\Lambda_{t} \otimes \mathrm{id}_{N}$ acting on the states $\rho^{S+S_{N}}$ of the system $S$ coupled with an arbitrary $N$-level system $S_{N}$, is also positive for all possible $N$.

If $\Lambda_{t}$ is only positive, troubles are expected when $S$ is coupled to a generic $N$-level system and the joint state $\rho^{S+S_{N}}$ carries correlations between $S$ and $S_{N}$. In fact, if $S$ and $S_{N}$ are not entangled, then $\rho^{S+S_{N}}=\rho^{S} \otimes \rho^{S_{N}}$ (or a convex combinations of factorized states), so that

$$
\Lambda_{t} \otimes \operatorname{id}_{N}\left[\rho^{S} \otimes \rho^{S_{N}}\right]=\Lambda_{t}\left[\rho^{S}\right] \otimes \rho^{S_{N}},
$$

and the positivity of $\Lambda_{t} \otimes \mathrm{id}_{N}$ automathically follows from the positivity of $\Lambda_{t}$.

However, let $S$ be a two-level system and $S_{N}=S_{2}=S$. As a common state $\rho^{S+S}$ we consider the projection

$$
\begin{aligned}
\rho_{A}= & \frac{1}{2}\left[\left(\begin{array}{ll}
1 & 0 \\
0 & 0
\end{array}\right) \otimes\left(\begin{array}{ll}
0 & 0 \\
0 & 1
\end{array}\right)+\left(\begin{array}{ll}
0 & 0 \\
0 & 1
\end{array}\right) \otimes\left(\begin{array}{ll}
1 & 0 \\
0 & 0
\end{array}\right)\right. \\
& \left.-\left(\begin{array}{ll}
0 & 1 \\
0 & 0
\end{array}\right) \otimes\left(\begin{array}{ll}
0 & 0 \\
1 & 0
\end{array}\right)-\left(\begin{array}{ll}
0 & 0 \\
1 & 0
\end{array}\right) \otimes\left(\begin{array}{ll}
0 & 1 \\
0 & 0
\end{array}\right)\right]
\end{aligned}
$$

onto a singlet-like state of $S+S$ with eigenvalues 0 and 1 . As a linear map on $S$, let us consider the transposition operation $T:\left(\begin{array}{ll}a & b \\ c & d\end{array}\right) \mapsto\left(\begin{array}{ll}a & c \\ b & d\end{array}\right)$. The map $T$ is positive, but

$$
\begin{aligned}
T \otimes \operatorname{id}_{2}\left[\rho_{A}\right]=\frac{1}{2} & {\left[\left(\begin{array}{ll}
1 & 0 \\
0 & 0
\end{array}\right) \otimes\left(\begin{array}{ll}
0 & 0 \\
0 & 1
\end{array}\right)+\left(\begin{array}{ll}
0 & 0 \\
0 & 1
\end{array}\right) \otimes\left(\begin{array}{ll}
1 & 0 \\
0 & 0
\end{array}\right)\right.} \\
& \left.-\left(\begin{array}{ll}
0 & 0 \\
1 & 0
\end{array}\right) \otimes\left(\begin{array}{ll}
0 & 1 \\
0 & 0
\end{array}\right)-\left(\begin{array}{ll}
0 & 1 \\
0 & 0
\end{array}\right) \otimes\left(\begin{array}{ll}
0 & 0 \\
1 & 0
\end{array}\right)\right]
\end{aligned}
$$


has eigenvalues $\pm 1 / 2$. Therefore the transposition $T$ is not completely positive, already the coupling to a 2-level system failing to be positive [5]. Clearly, the origin of troubles is the term $(2.2 \mathrm{~b})$ which encodes the entanglement between the two systems and is changed by $T \otimes \mathrm{id}_{2}$ into (2.3b). Instead, the term $(2.2 a)$, which represents an uncorrelated density matrix, is left unchanged.

Stinespring's decomposition theorem [11] ensures that the most general completely positive linear map $\Lambda$ on the states of $S$ is of the form

$$
\Lambda\left[\rho^{S}\right]=\sum_{\ell} V_{\ell}^{\dagger} \rho^{S} V_{\ell}
$$

where $V_{\ell}$ are suitable bounded operators. Evidently, $\Lambda \otimes \operatorname{id}_{N}\left[\rho^{S+S_{N}}\right]$ is positive for any positive $\rho^{S+S_{N}}$. The quantum mechanical time-evolution

$$
\Lambda_{t}\left[\rho^{S}\right]=\mathrm{e}^{-i t H} \rho^{S} \mathrm{e}^{i t H^{\dagger}}
$$

is of the form (2.4) and maps pure states into pure states thus preserving coherence. However, if one considers open quantum systems, coherence is usually lost. Hence, $\Lambda_{t}$ cannot be of the form (2.5); whether it is of the form (2.4) must be decided on physical grounds.

Remark 2.1 In the approach to $K$-mesons as open quantum systems [23-26], the transpositio $n$ map $T$ is replaced by a phenomenological dynamical map $\Lambda_{t}$ and, in [24,26], $T \otimes \operatorname{id}_{2}$ by $\Lambda_{t} \otimes \Lambda_{t}$. It can be shown $[26,27,29]$ that $\Lambda_{t}$ must be completely positive. Otherwise, physically realizable initial correlated states of two K-mesons as in (2.2) would develop negative eigenvalues.

\section{Reduced Dynamics}

The quantum open system of interest $S$ is assumed to be weakly interacting with a large (infinite) environment $R$, the dynamics of $S+R$ being governed by the Hamiltonian

$$
H=H_{S}+H_{R}+\lambda H_{S R}
$$

where $H_{S}$ and $H_{R}$ are the Hamiltonians of the system $S$, respectively environment $R$ and $H_{S R}$ is an interaction term with coupling strength $\lambda$. The total system $S+R$ is closed and its states, represented by density matrices $\rho$, evolve reversibly according to

$$
\frac{\partial \rho_{t}}{\partial t}=L_{H}\left[\rho_{t}\right]:=-i\left[H, \rho_{t}\right]
$$

The environment is supposed to be in equilibrium with respect to $H_{R}$, thus $\left[\rho^{R}, H_{R}\right]=0$. Let $R$ be a heat bath in equilibrium at temperature $\beta^{-1}$, namely we take

$$
\rho^{R}=\frac{\exp \left(-\beta H_{R}\right)}{\operatorname{Tr} \exp \left(-\beta H_{R}\right)} .
$$


The interaction term is chosen to be of the form

$$
H_{S R}=\sum_{a} A_{a}^{S} \otimes A_{a}^{R}
$$

where the self-adjoint operators $A_{a}^{S}$ and $A_{a}^{R}$ refer to the system and environment, respectively. It is no restrictive to assume $\varphi_{R}\left(A_{a}^{R}\right)=0$ for all $a$.

\section{Remarks 3.1}

i) The environment must eventually be considered infinite dimensional in order to allow for continuous spectra of $H_{R}$ and avoid recurrences. The states $\rho^{R}$ are thus not confined to density matrices, nor are the expectation values

$$
\varphi_{R}\left(A^{R}\right)=\operatorname{Tr}_{R}\left(\rho^{R} A^{R}\right)
$$

always expressible via a trace operation. However, we will stick to the density matrix notation, the genuine case of infinitely many degrees of freedom being easily retrieved [5-8].

ii) The dissipative effects perturb the time-evolution of $S$ given by its own Hamiltonian $H_{S}$ and are at least of second order in the coupling $\lambda$. The weak-coupling limit consists in going from the fast-time variable $t$ to the slow-time variable $\tau=\lambda^{2} t$, with $\lambda \rightarrow 0$. The technical procedure is physically justified when the ratio $\tau_{R} / \tau_{S}$ between the characteristic time $\tau_{R}$ of the environment and the characteristic time of the dissipative effects on $S, \tau_{S}$, is small [3]. However, there might be heat bath temperatures for which one has to retain higher powers in $\tau_{R} / \tau_{S}$, being forced to go beyond the weak-coupling limit [15].

In the interaction representation, $\widetilde{\rho}_{t}:=\exp \left(-t L_{0}\right)\left[\rho_{t}\right]$ with $L_{0}[\rho]:=-i\left[H_{S}+H_{R}, \rho\right]$, the evolution equation reads

$$
\frac{\partial \widetilde{\rho}_{t}}{\partial t}=\lambda \mathrm{e}^{-t L_{0}} L_{H_{S R}} \mathrm{e}^{t L_{0}}\left[\widetilde{\rho}_{t}\right]
$$

For the sake of simplicity, we assume the spectrum of $H_{S}$ to be discrete and nondegenerate with eigenvalues $\varepsilon_{r}$ and eigenvectors $|r\rangle$. We enumerate the operators $|r\rangle\langle s|$ by denoting them as $V_{j}^{S}$, so that $H_{S R}=\sum_{j} V_{j}^{S} \otimes V_{j}^{R}$ with $V_{j}^{R}=\sum_{a} \operatorname{Tr}_{S}\left(V_{j}^{S} A_{a}^{S}\right) A_{a}^{R}$. Further, setting $\omega_{j}:=\varepsilon_{r}-\varepsilon_{s}$, we get

$$
H_{S R}(t)=\mathrm{e}^{-t L_{0}}\left[H_{S R}\right]=\sum_{j} \mathrm{e}^{-i \omega_{j} t} V_{j}^{S} \otimes \mathrm{e}^{i t H_{R}} V_{j}^{R} \mathrm{e}^{-i t H_{R}}
$$

The formal solution of $(3.6)$ is $\widetilde{\rho}_{t}=\left(1+\sum_{n=1}^{\infty} \lambda^{n} U_{t}^{(n)}\right)\left[\widetilde{\rho}_{0}\right]$, with

$$
U_{t}^{(n)}=\int_{0}^{t} \mathrm{~d} t_{1} \int_{0}^{t_{1}} \mathrm{~d} t_{2} \cdots \int_{0}^{t_{n-1}} \mathrm{~d} t_{n} L_{H_{S R}\left(t_{1}\right)} L_{H_{S R}\left(t_{2}\right)} \cdots L_{H_{S R}\left(t_{n}\right)},
$$

where $L_{A}[\cdot]:=-i[A, \cdot]$. 
In order to extract the system $S$ reduced dynamics, we operate on the states $\rho$ of $S+R$ with the projector $P[\rho]=\operatorname{Tr}_{\mathrm{R}}(\rho) \otimes \rho_{R}$ which decouples the environment degrees of freedom. Further, we take as initial state of $S+R$ the state $\rho_{0}=\widetilde{\rho}_{0}=\rho^{S} \otimes \rho^{R}$ with no correlation between $S$ and $R$. It follows that $P\left[\rho_{0}\right]=\rho_{0}$.

Remark 3.2 Despite the fact that they are the most used [1-3], the choice of $P$ made above and the assumption on the initial global state cannot be generically upheld. In particular, one cannot always benefit from a factorized initial state. However, this is in many instances plausible, as in the case of neutral $K$-mesons in a gravitational background. Indeed, $K$-mesons produced in strong $\phi$-meson decays are arguably not influenced by geometrical fluctuations of gravitational origin . In general, one may be forced to adopt different projectors suited to initial states where system $S$ and environment $R$ result correlated by interactions prior $t=0[13-17,21]$.

We now elaborate more in detail on the approach of [19]. We assume the environment to be a Bose thermal bath described by the equilibrium state (3.3). The projector $P$ involves bath expectations with respect to (3.3), then only even correlation functions survive. Keeping terms up to $\lambda^{4}$, one eventually finds

$$
\frac{\partial P\left[\widetilde{\rho}_{t}\right]}{\partial t}=\lambda^{2} P \dot{U}_{t}^{(2)} P\left[\widetilde{\rho}_{t}\right]+\lambda^{4}\left[P \dot{U}_{t}^{(4)}-P \dot{U}_{t}^{(2)} P U_{t}^{(2)}\right] P\left[\widetilde{\rho}_{t}\right]
$$

where $\dot{U}_{t}^{(n)}$ is the time-derivative of $U^{(n)}$. In particular, the 2-nd and 4-th order contributions, $P \dot{U}_{t}^{(2)}$ and $P \dot{U}_{t}^{(2)} P U_{t}^{(2)}$ read

$$
\begin{aligned}
& P \dot{U}_{t}^{(2)}=\int_{0}^{t} \mathrm{~d} t_{1} P L_{H_{S R}(t)} L_{H_{S R}\left(t_{1}\right)} \\
& P \dot{U}_{t}^{(2)} P U_{t}^{(2)}=\int_{0}^{t} \mathrm{~d} t_{1} \int_{0}^{t} \mathrm{~d} t_{2} \int_{0}^{t_{2}} \mathrm{~d} t_{3} P L_{H_{S R}(t)} L_{H_{S R}\left(t_{1}\right)} P L_{H_{S R}\left(t_{2}\right)} L_{H_{S R}\left(t_{3}\right)} \cdot
\end{aligned}
$$

Remark 3.3 After standard rearrangement of the integrals in (3.10b), the whole 4th order contribution in (3.9) assumes a typical cumulant expression [19,20]. Thermal correlation functions are expected to factorize for large times; in such a case the cumulants vanish and allow one to operate a Markov approximation also at 4-th order in $\lambda$.

Since $\rho^{R}$ commutes with $H_{R}$, setting $\rho_{t}^{S}:=\operatorname{Tr}_{R}\left(\rho_{t}\right)$, the time-evolution equation obeyed by the open system $S$ has the form

$$
\frac{\partial \rho_{t}^{S}}{\partial t}=L_{H_{S}}\left[\rho_{t}^{S}\right]+\lambda^{2} K_{t}^{(2)}\left[\rho_{t}^{S}\right]+\lambda^{4} K_{t}^{(4)}\left[\rho_{t}^{S}\right]
$$

Let $\Omega_{j k}^{ \pm}(t):=\varphi_{R}\left(V_{j}^{R}( \pm t) V_{k}^{R}\right)$ denote the environment two-point correlation functions. Then, the 2 -nd order dissipative contribution in (3.9) explicitly reads

$$
K_{t}^{(2)}\left[\rho^{S}\right]=\sum_{j, k} \int_{0}^{t} \mathrm{~d} t_{1} \mathrm{e}^{-i t_{1} \omega_{j}}\left(\Omega_{k j}^{+}\left(t_{1}\right)\left[V_{j}^{S} \rho^{S}, V_{k}^{S}\right]+\Omega_{j k}^{-}\left(t_{1}\right)\left[V_{k}^{S}, \rho^{S} V_{j}^{S}\right]\right) .
$$


From (3.10b) it follows that, because of the chosen projector $P$, the 4 -th order dissipative operator $K_{t}^{(4)}$ involves four-point thermal correlation functions, which in turn are linear combinations of two-point ones. After a lengthy calculation one arrives at

$$
K_{t}^{(4)}\left[\rho^{S}\right]=\sum_{j, k, \ell, m} \sum_{p=1}^{10} \int_{0}^{t} \int_{0}^{t-t_{1}} \int_{0}^{t-t_{1}-t_{2}} \mathrm{~d} \vec{t} \mathrm{e}^{-i \vec{\Delta}_{k \ell m} \cdot \vec{t}} \Omega_{j k \ell m}^{(p)}(\vec{t}) D_{j k \ell m}^{(p)}\left[\rho^{S}\right],
$$

where $\vec{t}=\left(t_{1}, t_{2}, t_{3}\right)$ and $\vec{\Delta}_{k \ell m} \cdot \vec{t}=\left(\omega_{k}+\omega_{\ell}+\omega_{m}\right) t_{1}+\left(\omega_{\ell}+\omega_{m}\right) t_{2}+\omega_{m} t_{3}$.

The quantities $\Omega^{(p)}(\vec{t})$ are products of two point-correlation functions, while the operators $D_{j k \ell m}^{(p)}$ are essentially double commutators of observables of $S$. For instance, in the case $p=1$, one has

$$
\Omega_{j k \ell m}^{(1)}(\vec{t}):=\Omega_{j \ell}^{+}\left(t_{1}+t_{2}\right) \Omega_{k m}^{+}\left(t_{2}+t_{3}\right), \quad D_{j k \ell m}^{(1)}\left[\rho^{S}\right]:=\left[V_{j}^{S},\left[V_{k}^{S}, V_{\ell}^{S}\right] V_{m}^{S} \rho^{S}\right]
$$

Because of the explicit dependence of both $K_{t}^{(2)}$ and $K_{t}^{(4)}$ on time, the right hand side of (3.11) retains memory effects and does not generate a semigroup. However, a Markov approximation can be performed based on the following argument (see Remark 3.3). The two-point correlation functions $\varphi_{R}\left(V_{j}^{R}(t) V_{k}^{R}\right)$ are expected to factorize for $t$ larger than the correlation-time $\tau^{R}$ of the environment which is much shorter than the typical time for the dissipative effects being felt by the subsystem $S$. Since we assumed $\varphi_{R}\left(A_{a}^{R}\right)=0$, it follows that $\varphi_{R}\left(V_{j}^{R}(t) V_{k}^{R}\right)=0$ for $t>>\tau^{R}$. Therefore, for times $t>>\tau^{R}$, the

time-dependent dissipative operators $K_{t}^{(2)}$ and $K_{t}^{(4)}$ can be replaced by time-independent dissipative operators $K^{(2)}$ and $K^{(4)}$, by extending to infinity each time-integration in (3.12) and (3.13). For a rigorous approach to this kind of Markov approximation the reader is referred to $[4,6]$. Explicitly,

$$
\begin{aligned}
& K^{(2)}\left[\rho^{S}\right]:=\sum_{j, k}\left\{\widehat{\Omega}_{k j}^{+}\left(\omega_{j}\right)\left[V_{k}^{S} \rho^{S}, V_{j}^{S}\right]+\widehat{\Omega}_{j k}^{-}\left(\omega_{j}\right)\left[V_{k}^{S}, \rho^{S} V_{j}^{S}\right]\right\} \\
& K^{(4)}\left[\rho^{S}\right]:=\sum_{j, k, \ell, m} \sum_{p=1}^{10}, \widehat{\Omega}_{j k \ell m}^{(p)}\left(\vec{\Delta}_{k \ell m}\right) D_{j k \ell m}^{(p)}\left[\rho^{S}\right],
\end{aligned}
$$

where $\widehat{\Omega}_{j k}^{ \pm}(\omega):=\int_{0}^{\infty} \mathrm{d} t \mathrm{e}^{-i \omega t} \Omega_{j k}^{ \pm}(t)$ and

$$
\widehat{\Omega}_{j k \ell m}^{(p)}\left(\vec{\Delta}_{k \ell m}\right):=\int_{0}^{\infty} \mathrm{d} t_{1} \int_{0}^{\infty} \mathrm{d} t_{2} \int_{0}^{\infty} \mathrm{d} t_{3} \mathrm{e}^{-i \vec{\Delta}_{k \ell m} \cdot \vec{t}} \Omega_{j k \ell m}^{(p)}(\vec{t}) .
$$

\section{Non-interacting Open Quantum Systems}

Let the open system $S$ consist of two non-interacting systems $S_{1}$ and $S_{2}$ whose dynamics, disregarding for the moment the presence of the environment $R$, is governed by the 
Hamiltonian operators $H_{S_{1}}$ and $H_{S_{2}}$. Again, we assume the energies $\varepsilon_{a r}$ of $H_{S_{a}}, a=1,2$, to be discrete and non-degenerate and set $\omega_{a j}:=\varepsilon_{a r}-\varepsilon_{a s}$.

In absence of $R$, the system $S=S_{1}+S_{2}$ would evolve in time according to the Hamiltonian $H_{S}=H_{S_{1}} \otimes \mathrm{id}_{2}+\mathrm{id}_{1} \otimes H_{S_{2}}$. Instead, we suppose $S_{1,2}$ to interact weakly and independently with a thermal bath in the state (3.4). We chose an interaction term of the form

$$
H_{S R}=\sum_{j}\left(\left(V_{1 j}^{S} \otimes \mathrm{id}_{2}\right) \otimes V_{1 j}^{R}+\left(\mathrm{id}_{1} \otimes V_{2 j}^{S}\right) \otimes V_{2 j}^{R}\right) .
$$

with $\varphi_{R}\left(V_{a j}^{R}\right)=0$ for all $j, a=1,2$, and same coupling constant $\lambda$.

The analysis of the previous section can be repeated in this new context, the major difference being that, inserting (4.1) in (3.10) extra-indices appear identifying the system $S_{a}, a=1,2$, the various operators refer to. As a consequence, the dissipative operator in (3.12) becomes

$$
K_{t}^{(2)}\left[\rho^{S}\right]=\sum_{a, b=1}^{2} \sum_{j, k} \int_{0}^{t} \mathrm{~d} t_{1} \mathrm{e}^{-i \omega_{a j} t_{1}}\left\{\Omega_{b k ; a j}^{+}\left(t_{1}\right)\left[V_{a j}^{S} \rho^{S}, V_{b k}^{S}\right]+\Omega_{a j ; b k}^{-}\left(t_{1}\right)\left[V_{b k}^{S}, \rho^{S} V_{a j}^{S}\right]\right\}
$$

where $\Omega_{a j ; b k}^{ \pm}(t)=\varphi_{R}\left(V_{a j}^{R}( \pm t) V_{b k}^{R}\right)$ and, for sake of simplicity, $V_{a j}^{S}$ denotes either $V_{1 j}^{S} \otimes \mathrm{id}_{2}$ or $\operatorname{id}_{1} \otimes V_{2 j}^{S}$. In turn, the 4 -th order operator reads

$$
K_{t}^{(4)}=\sum_{\substack{a, b, c, d \\ j, k, l, m}} \sum_{p=1}^{10} \int_{0}^{t} \int_{0}^{t_{1}} \int_{0}^{t-t_{1}-t_{2}} \mathrm{~d} \vec{t} \mathrm{e}^{-i \vec{\Delta}_{b k ; c \ell ; d m} \cdot \vec{t}} \Omega_{a j ; b k ; c \ell ; d m}^{(p)}(\vec{t}) D_{a j ; b k ; c \ell ; d m}^{(p)},
$$

where $\vec{\Delta}_{b k ; \ell \ell ; m} \cdot \vec{t}=\left(\omega_{b k}+\omega_{c \ell}+\omega_{d m}\right) t_{1}+\left(\omega_{c \ell}+\omega_{d m}\right) t_{2}+\omega_{d m} t_{3}$ and

$$
\begin{aligned}
& \Omega_{a j ; b k ; c \ell ; d m}^{(1)}(\vec{t}):=\Omega_{a j ; c \ell}^{+}\left(t_{1}+t_{2}\right) \Omega_{b k ; d m}^{+}\left(t_{2}+t_{3}\right) \\
& D_{a j ; b k ; c \ell ; d m}^{(1)}\left[\rho^{S}\right]:=\left[V_{a j}^{S},\left[V_{b k}^{S}, V_{c \ell}^{S}\right] V_{d m}^{S} \rho^{S}\right] .
\end{aligned}
$$

The two-point correlation functions $\Omega_{a j ; b k}^{ \pm}(t)$ involve either bath operators interacting with the same system, $a=c$, or with different systems, $a \neq b$. We can perform the Markov approximation exactly as in the case of just one system $S$ so that

$$
\begin{aligned}
& K^{(2)}\left[\rho^{S}\right]:=\sum_{\substack{a, b \\
j, k}}\left\{\widehat{\Omega}_{b k ; a j}^{+}\left(\omega_{a j}\right)\left[V_{a j}^{S} \rho^{S}, V_{b k}^{S}\right]+\widehat{\Omega}_{a j ; b k}^{-}\left(\omega_{a j}\right)\left[V_{b k}^{S}, \rho^{S} V_{a j}^{S}\right]\right\} \\
& K^{(4)}\left[\rho^{S}\right]:=\sum_{\substack{a, b, c, d \\
j, k, \ell, m}} \sum_{p=1}^{10} \widehat{\Omega}_{a j ; b k ; c \ell ; d m}^{(p)}\left(\vec{\Delta}_{b k ; c \ell ; d m}\right) D_{a j ; b k ; c \ell ; d m}^{(p)}\left[\rho^{S}\right],
\end{aligned}
$$

where $\widehat{\Omega}_{a j ; b k}^{ \pm}(\omega):=\int_{0}^{\infty} \mathrm{d} t \mathrm{e}^{-i \omega t} \Omega_{a j ; b k}^{ \pm}(t)$ and

$$
\widehat{\Omega}_{a j ; b k ; c \ell ; d m}^{(p)}\left(\vec{\Delta}_{b k ; c \ell ; d m}\right):=\int_{0}^{\infty} \mathrm{d} t_{1} \int_{0}^{\infty} \mathrm{d} t_{2} \int_{0}^{\infty} \mathrm{d} t_{3} \mathrm{e}^{-i \vec{\Delta}_{b k ; c \ell ; d m} \cdot \vec{t}} \Omega_{a j ; b k ; c \ell ; d m}^{(p)}(\vec{t}) .
$$


Using (4.1) and (3.7), the 2-nd order dissipative operator can be written as follows:

$$
\begin{aligned}
K^{(2)}\left[\rho^{S}\right] & =K_{1}^{(2)} \otimes \operatorname{id}_{2}\left[\rho^{S}\right]+\operatorname{id}_{1} \otimes K_{2}^{(2)}\left[\rho^{S}\right] \\
& +\sum_{\substack{a \neq b \\
j, k}}\left\{\widehat{\Omega}_{b k ; a j}^{+}\left(\omega_{a j}\right)\left[V_{a j}^{S} \rho^{S}, V_{b k}^{S}\right]+\widehat{\Omega}_{a j ; b k}^{-}\left(\omega_{a j}\right)\left[V_{b k}^{S}, \rho^{S} V_{a j}^{S}\right]\right\} .
\end{aligned}
$$

The terms $K_{a}^{(2)}, a=1,2$ in (4.7a) are dissipative operators of the form (3.15a), involving only observables referring to the system $S_{a}$. If the contribution (4.7b) were absent, the right hand side of (4.7a) would generate a factorized time-evolution $\rho^{S} \mapsto \rho_{t}^{S}=\Lambda_{t}^{1} \otimes \Lambda_{t}^{2}\left[\rho^{S}\right]$, with $\Lambda_{t}^{1}$ satisfying

$$
\frac{\partial}{\partial t}\left(\Lambda_{t}^{1} \otimes \operatorname{id}_{2}\left[\rho^{S}\right]\right)=K_{1}^{(2)} \otimes \operatorname{id}_{2}\left[\Lambda_{t}^{1} \otimes \operatorname{id}_{2}\left[\rho^{S}\right]\right]
$$

and analogously for $\Lambda_{t}^{2}$.

Clearly, the term (4.7b) dynamically couples the two systems $S_{1}$ and $S_{2}$ through their interaction with the same environment. However, this coupling depends on the strength of the thermal correlations between bath operators describing the interaction with different subsystems. If there are no correlatoins between them, that is if

$$
\Omega_{a j ; c \ell}^{ \pm}(t)=\varphi_{R}\left(V_{a j}^{R}( \pm t)\right) \varphi_{R}\left(V_{c \ell}^{R}\right),
$$

whenever $a \neq c$ and $t>0$, then the term (4.7b) does not contribute since, with no restriction, we can assume the one-point bath correlation functions to vanish.

The same result follows from (4.9) when we include 4-th order dissipative effects. This can be seen as follows. In the expression (4.4b), the right hand side vanishes if $b \neq c$, since then $V_{b k}^{R}$ and $V_{c l}^{R}$ belong to different subsystems and thus commute. With $b=c$, using (4.9) one sees that the right hand side of (4.4a) vanishes unless $a=c=b=d$. Thus, if (4.9) holds, only $a=b=c=d=1$ and $a=b=c=d=2$ contribute to the sum in (4.5b). Then the 4 -th order dissipative operator splits as the 2-nd order one,

$$
K^{(4)}\left[\rho^{S}\right]=K_{1}^{(4)} \otimes \operatorname{id}_{2}\left[\rho^{S}\right]+\operatorname{id}_{1} \otimes K_{2}^{(4)}\left[\rho^{S}\right],
$$

where

$$
K_{a}^{(4)}=\sum_{j, k, \ell, m} \sum_{p=1}^{10} \widehat{\Omega}_{a j ; a k ; a \ell ; a m}^{(p)}\left(\vec{\Delta}_{a k ; a \ell ; a m}\right) D_{a j ; a k ; a \ell ; a m}^{(p)}, \quad a=1,2 .
$$

As a consequence of (4.9), the evolution equation (3.11), after the Markov approximation, becomes

$$
\partial_{t} \rho_{t}^{S}=\left(\left(L_{H_{S_{1}}}+K_{1}^{(2)}+K_{1}^{(4)}\right) \otimes \mathrm{id}_{2}+\mathrm{id}_{1} \otimes\left(L_{H_{S_{2}}}+K_{2}^{(2)}+K_{2}^{(4)}\right)\right)\left[\rho_{t}^{S}\right]
$$


and the dynamical maps generated by it factorize into $\Lambda_{t}^{1} \otimes \Lambda_{t}^{2}$, where $\Lambda_{t}^{1}$ and $\Lambda_{t}^{2}$ are generated as in (4.8) with $K_{a}^{(2)}, a=1,2$, replaced by $K_{a}^{(2)}+K_{a}^{(4)}$.

\section{Conclusions}

The request that the reduced dynamics of open quantum systems in interaction with a reservoir be completely positive is often rejected as not physically necessary [12-14,16-21]; it lacks physical appeal and involves trivial and uncontrollable couplings of the systems with generic $N$-level systems.

The issue of complete positivity assumes its full physical significance when dealing with the dynamics of correlated systems interacting with an environment. All depends on whether the reduced dynamics of the subsystems factorizes as $\Lambda_{t} \otimes \Lambda_{t}$, thus indicating that they evolve independently from each other, remaining dynamically uncorrelated.

If this is the case, then the complete positivity of the single system reduced dynamics $\Lambda_{t}$ is unescapable, otherwise the joint reduced dynamics $\Lambda_{t} \otimes \Lambda_{t}$ generates unacceptable negative probabilities. ¿From this point of view, the necessity of complete positivity appears to be a dynamical aspect of quantum entanglement.

On the other hand, there are situations for which the factorization of the reduced dynamics is not the case due to the subsystem-environment interaction and to the physics of the environment itself, namely the behaviour of its correlation functions. If the twosystem reduced dynamics does not factorize, it is not compelling that the single-system reduced dynamics be completely positive.

In the literature there is evidence of non-completely positive reduced dynamics beyond the weak-coupling limit [17-20], namely taking into account contributions of order $\lambda^{4}$ in the coupling between subsystem and environment. We have shown that if the reduced dynamics of two non-interacting subsystems factorizes at 2-nd order in $\lambda$, it factorizes also at 4-th order. Therefore, absence of complete positivity in the single-system reduced dynamics at 4-th order would jeopardize the description of the physical behaviour of two of these subsystems at the same order of approximation.

Finally, we would like to stress that there are physical instances where an experimental check of complete positivity seems achievable, as in the case of $K$-mesons [25-29]; there, the conditions for a factorized reduced dynamics are plausibly fulfilled because of the weakness of the effects of the gravitational background. The reduced dynamics of $K$-mesons as open quantum systems must then be completely positive and experiments at $\phi$-factories can explicitly clarify this fundamental request. 


\section{REFERENCES}

1. E.B. Davies, Quantum Theory of Open Systems, (Academic Press, New York, 1976)

2. H. Spohn, Rev. Mod. Phys. 53 (1980) 569

3. R. Alicki and K. Lendi, Quantum Dynamical Semigroups and Applications, Lect. Notes Phys. 286, (Springer-Verlag, Berlin, 1987)

4. R. Dumcke and H. Spohn, Z. Physik B34 (1979) 419

5. V. Gorini, A. Kossakowski and E.C.G. Surdarshan, J. Math. Phys. 17 (1976) 821

6. V. Gorini, A. Frigerio, M. Verri, A. Kossakowski and E.C.G. Surdarshan, Rep. Math. Phys. 13 (1978) 149

7. V. Gorini and A. Kossakowski, J. Math. Phys. 17 (1975) 1298

8. A. Frigerio and V. Gorini, J. Math. Phys. 17 (1976) 2123

9. G. Lindblad, Commun. Math. Phys. 48 (1976) 119

10. M. Takesaki, Theory of Operator Algebras I (Springer, Berlin, 1979)

11. K. Kraus, States, Effects and Operations, Lecture Notes in Physics 190 (Springer, Belrin, 1983)

12. P. Pechukas, Phys. Rev. Lett. 73 (1994) 1060

13. V. Romero-Rochin and I. Oppenheim, Physica A 155 (1988) 52

14. V. Romero-Rochin, A. Orsky and I. Oppenheim, Physica A 156 (1989) 244

15. V. Gorini, M. Verri and A. Frigerio, Physica A 161 (1989) 357

16. A. Suarez, R. Silbey and I. Oppenheim, J. Chem. Phys. 97 (1992 ) 5101

17. T.-M. Chang and J.L. Skinner, Physica A 193 (1993) 483

18. J. Budimir and J.L. Skinner, J. Stat. Phys. 49 (1987) 1029

19. B.B. Laird, J. Budimir and J.L. Skinner, J. Chem. Phys. 94 (1991) 4391

20. B.B. Laird and J.L. Skinner, J. Chem. Phys. 94 (1991) 4405

21. A. Royer, Phys. Rev. Lett. 77 (1996) 3272

22. C.P. Slichter, Principles of Magnetic Resonance, with Examples from Solid State Physics (Springer, Berlin, New York, 1990)

23. J. Ellis, J.S. Hagelin, D.V. Nanopoulos and M. Srednicki, Nucl. Phys. B241 (1984) 381 ;

24. P. Huet and M.E. Peskin, Nucl. Phys. B434 (1995) 3

25. F. Benatti and R. Floreanini, Nucl. Phys. B488 (1997) 335

26. F. Benatti and R. Floreanini, Nucl. Phys. B511 (1998) 550

27. F. Benatti and R. Floreanini, Mod. Phys. Lett. A12 (1997) 1465

28. F. Benatti and R. Floreanini, Banach Centre Publ. 43 (1998) 71

29. F. Benatti and R. Floreanini, Phys. Lett. B 468 (1999) 287 